\title{
Machine Learning and Machine Vision Accelerate 3D Printed Orodispersible Film Development
}

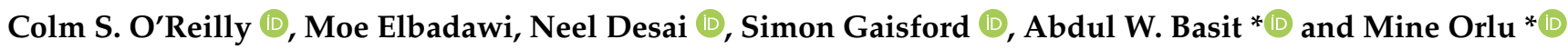 \\ Department of Pharmaceutics, UCL School of Pharmacy, University College London, 29-39 Brunswick Square, \\ London WC1N 1AX, UK; colm.o'reilly.17@ucl.ac.uk (C.S.O.); m.elbadawi@ucl.ac.uk (M.E.); \\ neel.desai.13@ucl.ac.uk (N.D.); s.gaisford@ucl.ac.uk (S.G.) \\ * Correspondence: a.basit@ucl.ac.uk (A.W.B.); m.orlu@ucl.ac.uk (M.O.)
}

Citation: O’Reilly, C.S.; Elbadawi, M.; Desai, N.; Gaisford, S.; Basit, A.W.; Orlu, M. Machine Learning and Machine Vision Accelerate 3D Printed Orodispersible Film Development. Pharmaceutics 2021, 13, 2187. https://doi.org/10.3390/ pharmaceutics13122187

Academic Editor: Werner Weitschies

Received: 14 November 2021 Accepted: 13 December 2021 Published: 17 December 2021

Publisher's Note: MDPI stays neutral with regard to jurisdictional claims in published maps and institutional affiliations.

Copyright: (C) 2021 by the authors Licensee MDPI, Basel, Switzerland. This article is an open access article distributed under the terms and conditions of the Creative Commons Attribution (CC BY) license (https:// creativecommons.org/licenses/by/ $4.0 /)$.

\begin{abstract}
Orodispersible films (ODFs) are an attractive delivery system for a myriad of clinical applications and possess both large economical and clinical rewards. However, the manufacturing of ODFs does not adhere to contemporary paradigms of personalised, on-demand medicine, nor sustainable manufacturing. To address these shortcomings, both three-dimensional (3D) printing and machine learning (ML) were employed to provide on-demand manufacturing and quality control checks of ODFs. Direct ink writing (DIW) was able to fabricate complex ODF shapes, with thicknesses of less than $100 \mu \mathrm{m}$. ML algorithms were explored to classify the ODFs according to their active ingredient, by using their near-infrared (NIR) spectrums. A supervised model of linear discriminant analysis was found to provide $100 \%$ accuracy in classifying ODFs. A subsequent partial least square algorithm was applied to verify the dose, where a coefficient of determination of $0.96,0.99$ and 0.98 was obtained for ODFs of paracetamol, caffeine, and theophylline, respectively. Therefore, it was concluded that the combination of 3D printing, NIR and ML can result in a rapid production and verification of ODFs. Additionally, a machine vision tool was used to automate the in vitro testing. These collective digital technologies demonstrate the potential to automate the ODF workflow.
\end{abstract}

Keywords: artificial intelligence; industry 4.0; additive manufacturing; thin film manufacture; personalized pharmaceuticals; semi-solid extrusion (SSE); computer vision; drug-loaded systems; digital pharmaceutics \& digital medicine; mobile 3D printing drug products

\section{Introduction}

Orodispersible films (ODFs) are an attractive delivery system, with clinically desirable features including high patient acceptability, requires no special administration instructions, and high drug loading [1,2]. For patients that have difficulty in swallowing, such as paediatric, geriatric and psychiatric patients, ODFs provide low risk of choking in comparison to conventional dosage forms [3]. Understandably, the market size is expected to reach USD 15.9 billion by the end of 2024, highlighting their economical and clinical rewards [4].

Despite the aforementioned benefits, both research and manufacturing of ODFs can be improved to enhance both clinical and manufacturing outcomes. For one, ODFs are batch manufactured, neglecting the current clinical paradigm of personalised medicines [5]. Conventional manufacturing methods of ODFs include solvent casting and film casting, where a bulk sheet of film is made and subsequently cut into the desired shapes. This postfabrication process lacks sustainability for three reasons: (i) it requires a post-processing stage of cutting the films into size; (ii) materials are subsequently discarded, resulting in material wastage; and (iii) as the films are made in one bulk, more time is needed for drying. Hence, conventional casting techniques are time consuming processes and produce unnecessary material waste, which also makes them undesirable for clinical settings. Fortunately, these limitations can be addressed by three-dimensional (3D) printing. Three-dimensional printing is an emerging fabrication technology that has been recently demonstrated to produce bespoke, complex delivery systems with digital precision [6-12]. 
For ODFs, 3D printing can produce personalised dosage and only print the desired shape, resulting in faster drying times, no material wastage and considerably reduced manual labour in the post-fabrication stage [13-19]. Moreover, 3D printing is also an attractive candidate for on-demand medicine manufacturing, owing to their compact, versatile, and user-friendly attributes [20-24].

While 3D printing is more promising and autonomous than conventional ODF fabrication methods, there are several bottlenecks that need to be addressed before the technology is transferred to clinical settings. Despite its superior autonomy, there remains a trial-anderror approach to making delivery systems via $3 \mathrm{D}$ printing, which can be time-consuming. In addition, the prospect of transferring 3D printing into clinics can be improved by the introduction of real time release (RTR) testing. RTR testing is the ability to evaluate and ensure the quality of in-process and/or final product based on process data [25]—one example would be the combination of vibrational spectroscopy (e.g., near infrared (NIR) spectroscopy) and chemometrics as an alternative for conventional quality control [26,27]. NIR spectroscopy is a fast, non-destructive, operator-friendly, and portable method that could facilitate the identification of drugs and quantification of dose in dosage forms and can be integrated at the point of dispensing. Trenfield et al., (2020) demonstrated the potential of NIR spectroscopy as a non-destructive quality control method where they used a point-and-shoot approach to quantify the amount of paracetamol in printlets [28]. The final piece of the puzzle is the interpretation of the NIR spectra which is not something that would be feasible in clinical settings due to time constraints and lack of NIR spectroscopy expertise. Machine learning (ML) may provide the answer to this final barrier.

ML is a subset of Artificial Intelligence (AI) that builds predictive or decision-making models using historical data and improves upon these models through experience [29-32]. Like 3DP, ML is impacting several sectors [33-35], such as outperforming clinicians in diagnosis in the medical sector [36] and streamlining the drug to market process in the pharmaceutical sector $[37,38]$. However, the concurrent use of ML in 3DP of pharmaceuticals is a largely untapped resource as a decision-making tool in formulation development. While recent research has investigated the use of ML to address the trial-and-error approach of $3 \mathrm{D}$ printing [39,40], the RTR workflow remains underexplored.

The present study demonstrates the successful integration of both 3D printing and ML to automate the ODF fabrication workflow. To rapidly produce personalised ODF shapes, 3D printing was employed. Thereafter, the ODF prints were verified for both drug and dose using a combination of NIR and ML. The model drugs selected for this study were paracetamol, caffeine, and theophylline. The latter two are structurally similar, and hence were used to 'stress test' the ML models. Lastly, an additional digital technology, machine vision (MV), was also employed to analyse the in vitro disintegration of films. $\mathrm{MV}$ is also a subset of AI that captures visual information via a camera and converts it into digital information to be processed. The study discusses the potential of all three digital technologies to automate the research workflow and help facilitate the transition of 3D printing into clinical settings.

\section{Materials and Methods}

\subsection{Materials}

Blanose Carboxymethyl Cellulose Type 7HF-PH (725 kDa) was provided by Ashland (Wilmington, DE, USA). Acetaminophen (paracetamol) powder (A5000-1KG), caffeine powder (W222402-1KG-K), theophylline powder (T1633-1KG), and Patent Blue V sodium salt for microscopy (21605-25G) were purchased from Sigma Aldrich (Gillingham, UK).

\subsection{Methods}

\subsubsection{Feedstock Formulation}

$\mathrm{CMC}$ feedstock formulations were prepared by adding the required amount of $\mathrm{CMC}$ slowly to $100 \mathrm{~mL}$ of deionised water in a beaker while stirring with a spatula at room temperature. The dispersion was left to stir for an hour on a hotplate stirrer with a magnetic 
stirrer rod to allow complete dissolution of the polymer and to allow dispersal of air bubbles. All mixing was performed at ambient temperature. For oral cavity model (OCM) analysis, formulations were dyed to visualise film dissolution by adding approximately $3 \mathrm{~mL}$ of $0.2 \%$ $w / v$ Patent Blue to $100 \mathrm{~mL}$ of formulation. For NIR and ML analysis, CMC formulations were loaded with API (paracetamol or caffeine or theophylline) by adding the required amount of API to deionised water and allowing complete dissolution of the drug and then adding the required amount of polymer. Amount of API to achieve a certain $\% w / w$ film was calculated by assuming all water would be removed by the drying process, as shown in Table 1.

Table 1. Formulae for drug-loaded feedstock formulations.

\begin{tabular}{cccc}
\hline Feedstock Formulation & $\begin{array}{c}\text { Water } \\
(\mathbf{m L})\end{array}$ & $\begin{array}{c}\text { API } \\
(\mathbf{g})\end{array}$ & $\begin{array}{c}\text { CMC } \\
(\mathbf{g})\end{array}$ \\
\hline $5 \% w / w$ Paracetamol & 100 & 0.13 & 2.5 \\
$10 \% w / w$ Paracetamol & 100 & 0.28 & 2.5 \\
$20 \% w / w$ Paracetamol & 100 & 0.625 & 2.5 \\
$5 \% w / w$ Caffeine & 100 & 0.13 & 2.5 \\
$10 \% w / w$ Caffeine & 100 & 0.28 & 2.5 \\
$20 \% w / w$ Caffeine & 100 & 0.625 & 2.5 \\
$5 \% w w$ Theophylline & 100 & 0.13 & 2.5 \\
$10 \% w / w$ Theophylline & 100 & 0.28 & 2.5 \\
$20 \% w$ Theophylline & 100 & 0.625 & 2.5 \\
\hline
\end{tabular}

\subsubsection{D Printing Process}

Onshape (Onshape Inc., Boston, MA, USA) was used to design the films used in this study. Figure 1 shows examples of each film design and image after printing, whereas representative images of drug-loaded films can be found in Figure S1. Designs were exported as stereolithography (.stl) files to the BioX Printer (Cellink, Gothenburg, Sweden), a direct-ink writing 3D printer, using a USB drive. Scotch Blue Trim and Baseboard's Painter's Tape \#2093EL was applied to the petri dish prior to printing. Table 2 shows optimised printing parameters which were selected based on initial 3D printing process development.

Table 2. Optimised printing parameters used in this study.

\begin{tabular}{cc}
\hline & Parameters \\
\hline Needle gauge (Needle diameter) & $22 \mathrm{G}(0.410 \mathrm{~mm})$ \\
Compressed air pressure & $100 \mathrm{kPa}$ \\
Printing speed & $20 \mathrm{~mm} / \mathrm{s}$ \\
Infill pattern & Grid infill \\
Infill density & $25 \%$ \\
\hline
\end{tabular}

Feedstock formulations were loaded into syringes then needle and compressed air line was attached to the syringe. Automatic bed levelling and calibration were performed to ensure uniform printing between batches. Once printed, films dried overnight at ambient conditions and then transferred to desiccator overnight to facilitate final drying. Thickness of films were measured on several locations on the films using a thickness gauge (Mercer Ltd., Manchester, UK). For OCM samples, $2.5 w / v \%$ CMC were printed with a blue dye. For NIR and ML analysis, drug-loaded feedstocks from Table 1 were printed using the parameters in Table 2. 

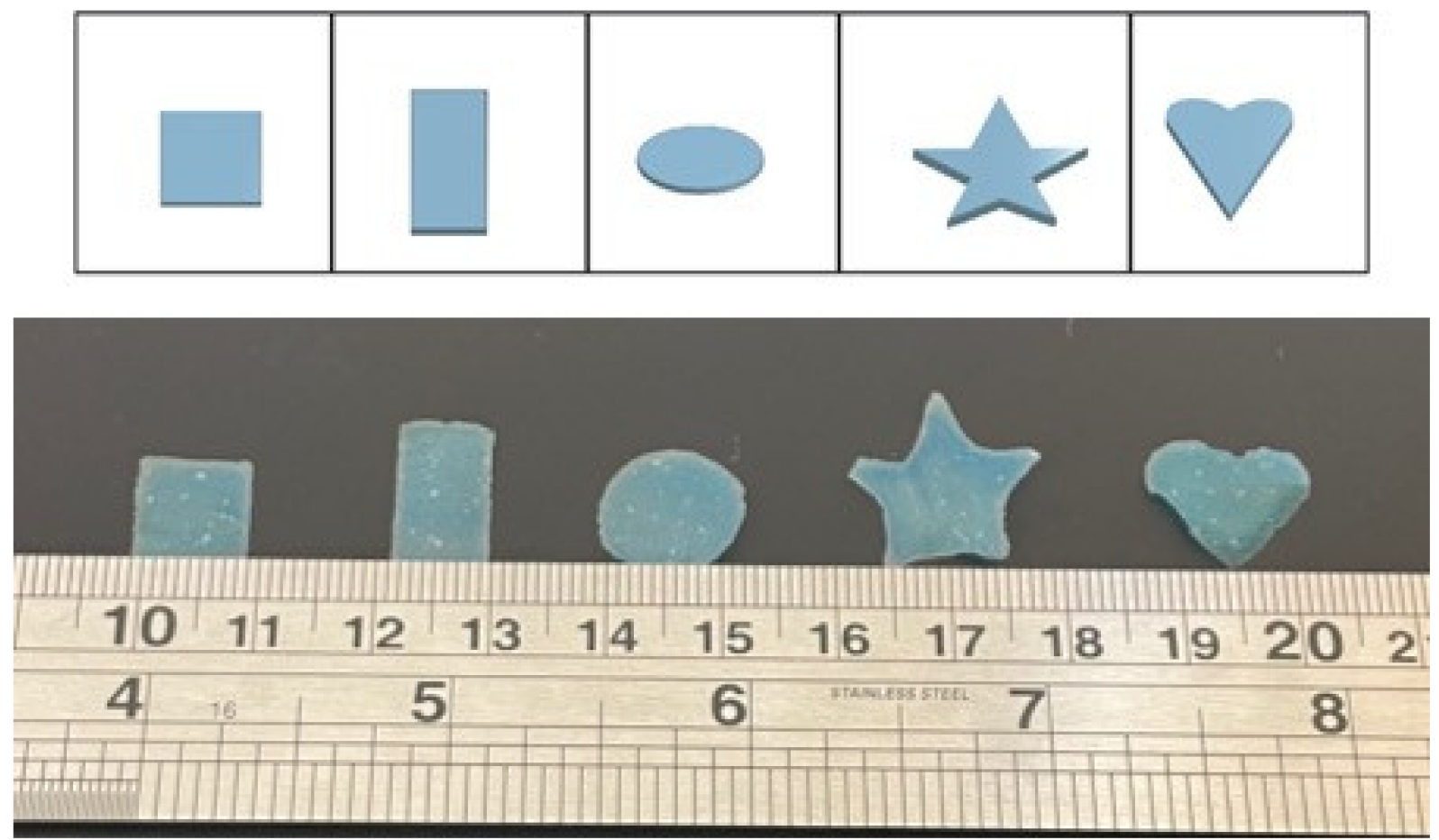

Figure 1. Examples of CAD designs of shapes (square, strip, circle, star, heart) used in this study (top) and examples of 3DP printed ODFs corresponding to the CAD designs (bottom) (Ruler markings in centimetres).

\subsubsection{Rheology}

Rheology profiles of the feedstock formulations were gathered via rotational rheometry using a Bohlin Gemini HR Nano (Malvern Instruments, Malvern, UK) equipped with a $4^{\circ} / 40$ Cone, with a gap of $150 \mu \mathrm{m}$. Samples were subjected to 25 different shear rates ascending logarithmically from 0.005 to $100 \mathrm{~s}^{-1}$ at $20^{\circ} \mathrm{C}$.

\subsubsection{Oral Cavity Model (OCM) Disintegration Testing}

The OCM facilitates physiologically relevant disintegration testing by simulating the swallowing processes observed in the human mouth and was developed using information from various images of the human buccal cavity and other data [41]. Swallowing, and thus disintegration, is achieved through cyclic compression cycles of a silicone tongue against an acrylic ceiling, with simulated salivary fluid (SSF) introduced into the OCM from the anterior to posterior direction at a rate of $1.5 \mathrm{~mL} / \mathrm{min}$ using a syringe driver [42].

The disintegration time was analysed using an MV algorithm. ODFs were placed in the middle of the tongue surface before starting the compression sequence. A mobile phone (Apple iPhone X, Apple Inc., Cupertino, CA, USA) was positioned on top of the acrylic plate with the camera facing down recording at 30 frames per second (fps). A single frame was extracted from the video between the compression and decompression phases and an area was calculated by identifying the perimeter of the ODF using MATLAB (MathWorks, Natick, MA, USA). For each ODF sample type tested mean and standard deviation of areas were calculated using built-in MATLAB functions $(n=3)$.

\subsubsection{Petri Dish Method Disintegration}

ODF samples were placed in $90 \mathrm{~mm}$ petri dishes, which were positioned between springs of a water bath with shaker functionality $\left(37^{\circ} \mathrm{C}, 70\right.$ shakes $\left./ \mathrm{min}\right)$. An amount of $2.5 \mathrm{~mL}$ of SSF was added directly onto samples in each petri dish. Disintegration times were recorded via stopwatch, and the point of disintegration established via operator observations (point at which structural integrity was lost). For each ODF type, $n=3$ samples were tested. 


\subsubsection{Near-Infrared Spectroscopy Analysis}

The near infrared spectra were collected using a MPA Bruker FT-NIR spectrophotometer (Bruker, Global). Pure CMC, paracetamol, caffeine, and theophylline powder were used and were measured as references; $n=6$ of each drug-loaded ODF type were scanned at a resolution of $1 \mathrm{~cm}^{-1}$ over a range of 12,000-4000 $\mathrm{cm}^{-1}$ for 64 scans, using a polytetrafluoroethylene (PTFE) backer.

\subsubsection{High-Performance Liquid Chromatography Analysis}

High-performance liquid chromatography (HPLC; Agilent 1260 Infinity II, Agilent Technologies, Santa Clara, CA, USA) was used to verify drug dose. Calibration curve was generated covering the concentration range of the films, 0.01 to $2.5 \mathrm{mg} / \mathrm{mL}$. The HPLC parameters for analysis of each drug varied and are enumerated in Table 3. An initial stock solution was prepared for each drug by pouring the drug into a $250 \mathrm{~mL}$ volumetric flask with the designated solution (Table 3). The stock solution was stirred using a magnetic stirrer until the powder completely dissolved. Working solutions were formed from the stock solution in $20 \mathrm{~mL}$ volumetric vials and diluted using the designated solution. For each solution, 50-60 $\mu \mathrm{L}$ was extracted using a syringe equipped with a $0.22 \mu \mathrm{m}$ PTFE filter and subsequently poured into HPLC vials. The working solutions were used to generate the calibration curve. For analysing the drug-loaded films, the same protocol was followed. First, the weight of each film was recorded. Then, the films were placed in a $5 \mathrm{~mL}$ flask and subsequently mixed with the designated solution until complete film disintegration. Thereafter, the films were filtered and analysed via the HPLC.

Table 3. HPLC parameters.

\begin{tabular}{cccc}
\hline Parameters & Paracetamol & Caffeine & Theophylline \\
\hline & A: Distilled Water & A: Orthophosphoric Acid & A: Distilled Water \\
B: Acetonitrile & C: Ethanol \\
Mobile phase composition & B: Methanol & & $60: 10: 30$ \\
Mobile Phase ratio & $85: 15$ & $80: 20$ & 1 \\
Flow rate $(\mathrm{mL} / \mathrm{min})$ & 1 & 1 & 1 \\
Injection volume $(\mu \mathrm{L})$ & 20 & 10 & 272 \\
Detection wavelength $(\mathrm{nm})$ & 247 & Luna C18 & Luna C18 \\
Column & Luna C18 & $(250 \times 4.6 \mathrm{~mm} ; 5 \mu \mathrm{m})$ & $(250 \times 4.6 \mathrm{~mm} ; 5 \mu \mathrm{m})$ \\
Column temperature $\left({ }^{\circ} \mathrm{C}\right)$ & $4.6 \mathrm{~mm} ; 5 \mathrm{~m})$ & 40 & 40 \\
Retention time $(\mathrm{mins})$ & 40 & 13.0 & 13.3 \\
\hline
\end{tabular}

\subsubsection{Machine Learning}

ML models were developed using Python (version 3.9, Wilmington, DE, USA) to identify the drug and dose of API in the ODFs, using the scikit-learn package (version 0.17.1). Unsupervised learning using Principal component analysis (PCA) on the raw data was attempted to see if ML could inherently recognise the differences between groups without labelling. Subsequently, supervised learning using linear discriminant analysis (LDA) was applied to the data. LDA is a supervised alternative of PCA using labelled data which tries to maximise the distance between groups. For LDA, the data were first preprocessed to reduce signal noise using Savitzky-Golay filter (parameters: derivative $=0$; window size $=21$; and polynomial $=1$ st order), and then the model was trained using a training:testing split of 70:30 to see if the model could identify unseen data. The regression analysis was performed using partial least squares (PLS). A training:testing split of 75:25 was used, where a lower testing size was used to account for the smaller sample size used for regression analysis. 


\section{Results and Discussion}

\subsection{D Printing Method Development}

The present study sought to leverage digital technologies to automate, and in essence, simplify the ODF fabrication pipeline. CMC was chosen as the model polymer due to its pharmaceutical relevance. Several concentrations ranging from 1 to $2.5 w / v \%$ CMC were printed. The ideal concentration for printing was $2.5 \mathrm{w} / \mathrm{v} \%$, which was found to both extrude and maintain print integrity post-extrusion. In contrast, $1 \mathrm{w} / \mathrm{v} \%$ was found to extrude but subsequently spread, resulting in a distorted print shape. Rheological analysis (Figure 2) revealed that increasing the CMC concentration from 1 to $2.5 w / v \%$ increased the Newtonian plateau by two orders of magnitude, from $10^{0}$ to $10^{2} \mathrm{~Pa} . \mathrm{s}$, which is in agreement with previous work [43]. Moreover, all formulations demonstrated shearthinning properties, which is desirable in 3D printing [44,45]. Thus, it was deduced that the structural integrity of $2.5 w / v \%$ was due to its higher viscosity values at low shear rates.

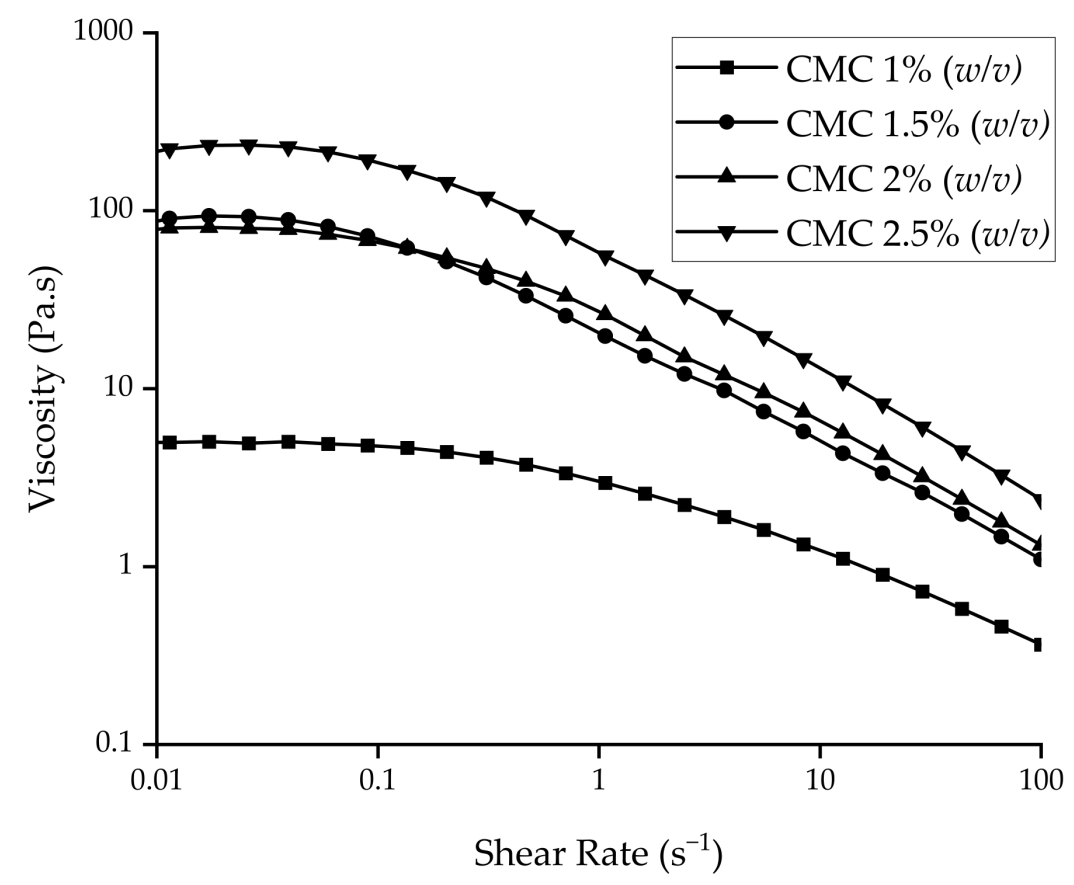

Figure 2. Rheology profiles of various concentrations of CMC feedstocks.

Further incorporation of materials, such as a dye for disintegration analysis or API, revealed minor increases in rheological properties to the selected CMC concentration. A dye was required for visualising the disintegration of films during OCM and MV analysis. The effects of the dye were negligible on the rheological profile, most likely due to the small amount of dye incorporated into the feedstocks, as illustrated in Figure 3A. The incorporation of APIs into $2.5 \mathrm{w} / \mathrm{v} \% \mathrm{CMC}$ concentration had minor effect on the viscosity (Figure 3B-D). The Newtonian plateau at low shear rates was found to remain in the order of $10^{2}$ Pa.s.

Based on these findings, formulations containing $2.5 w / \% \%$ CMC were used for subsequent analyses. The design and printing parameters were then optimised, where varying the infill density (ID) was found to affect the thickness of films. Strip designs resulted in film thicknesses of $73.34 \pm 0,95.56 \pm 10.72$ and $131.12 \pm 9.63 \mu \mathrm{m}$, with ID of $25 \%, 40 \%$ and $50 \%$, respectively. Thus, increasing the ID increased the film thickness. Strips with ID of $10 \%$ or below, and $50 \%$ or above were explored but these produced films with poor resolution. Hence, it was concluded that the ideal ID were $25 \%$ and $40 \%$. 
(A)

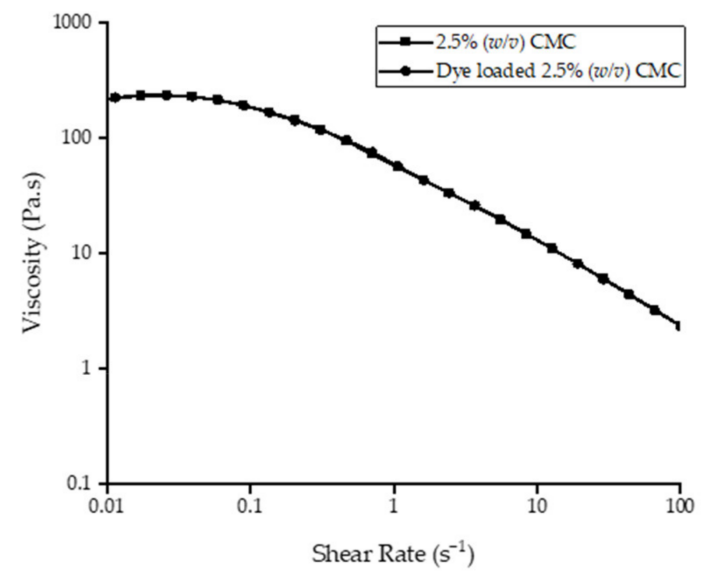

(C)

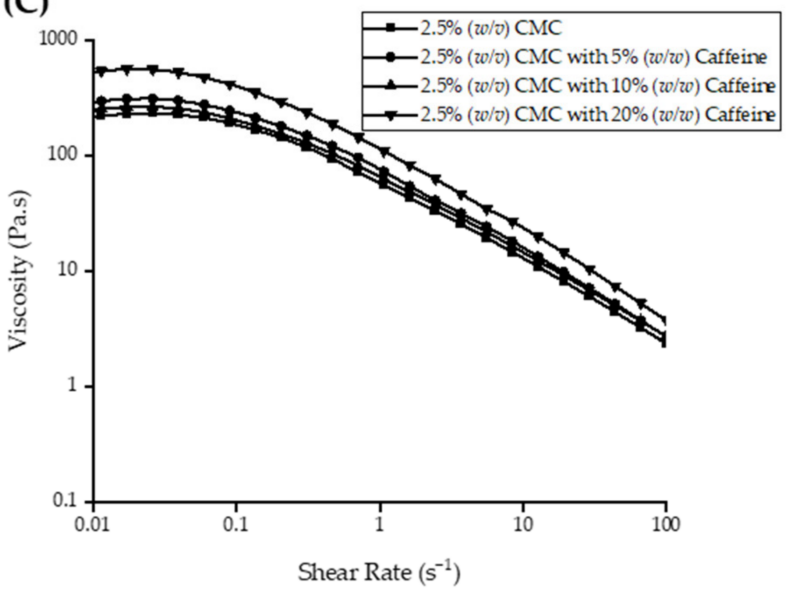

(B)

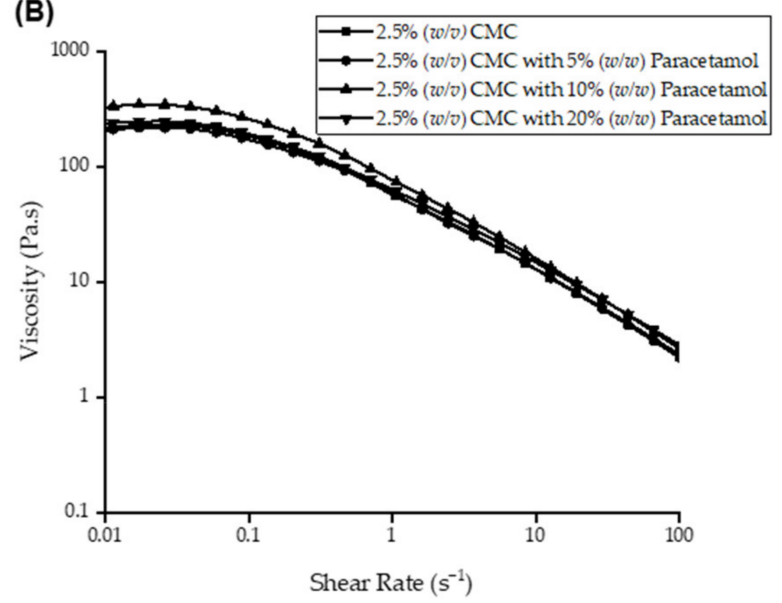

(D)

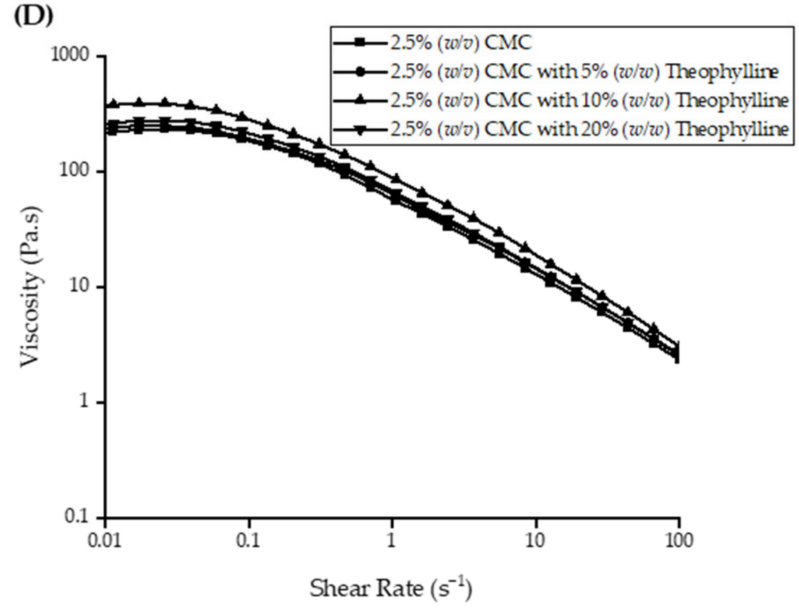

Figure 3. Rheological profiles of both (A) dye-loaded and (B-D) API-loaded $2.5 w / v \%$ CMC solutions.

\subsection{ODF Disintegration Study}

ODFs were assessed for their disintegration characteristics using the OCM. The data analysis process was automated via an MV algorithm. Strips with ID of $25 \%$ were found to disintegrate in under $180 \mathrm{~s}$ (Table 4) hence adhering to the Pharmacopeial requirement for suitable oral delivery [46], with a mean disintegration time of $80 \mathrm{~s}$ (Figure 4A). Strips with ID of $40 \%$ were less predictable when assessed using the OCM. These films remained intact for the first $90 \mathrm{~s}$, and consistently fell off the tongue thereafter whilst remaining intact. Thus, it was difficult to ascertain whether ID of $40 \%$ would pass the European Pharmacopeial requirement of $180 \mathrm{~s}$ (Figure 4B). It was concluded that polymeric solutions of $2.5 \mathrm{w} / \mathrm{v} \%$, with $25 \%$ ID, was suitable for oral delivery, whereby films with good resolution were obtained, and were able to disintegrate within the European Pharmacopeial requirements. To further validate this observation, films of star and circle geometries were also examined using the OCM. Once more, it was discovered that these films were able to adhere to the European Pharmacopeial requirements (Figure 5). The thickness of star and circle shaped films printed with an ID of $25 \%$ were measured as $63.34 \pm 5.78 \mu \mathrm{m}$ and $93.34 \pm 5.78 \mu \mathrm{m}$, respectively. 
Table 4. Extracted frames from videos showing ODF samples during disintegration in OCM (IDinfill density).

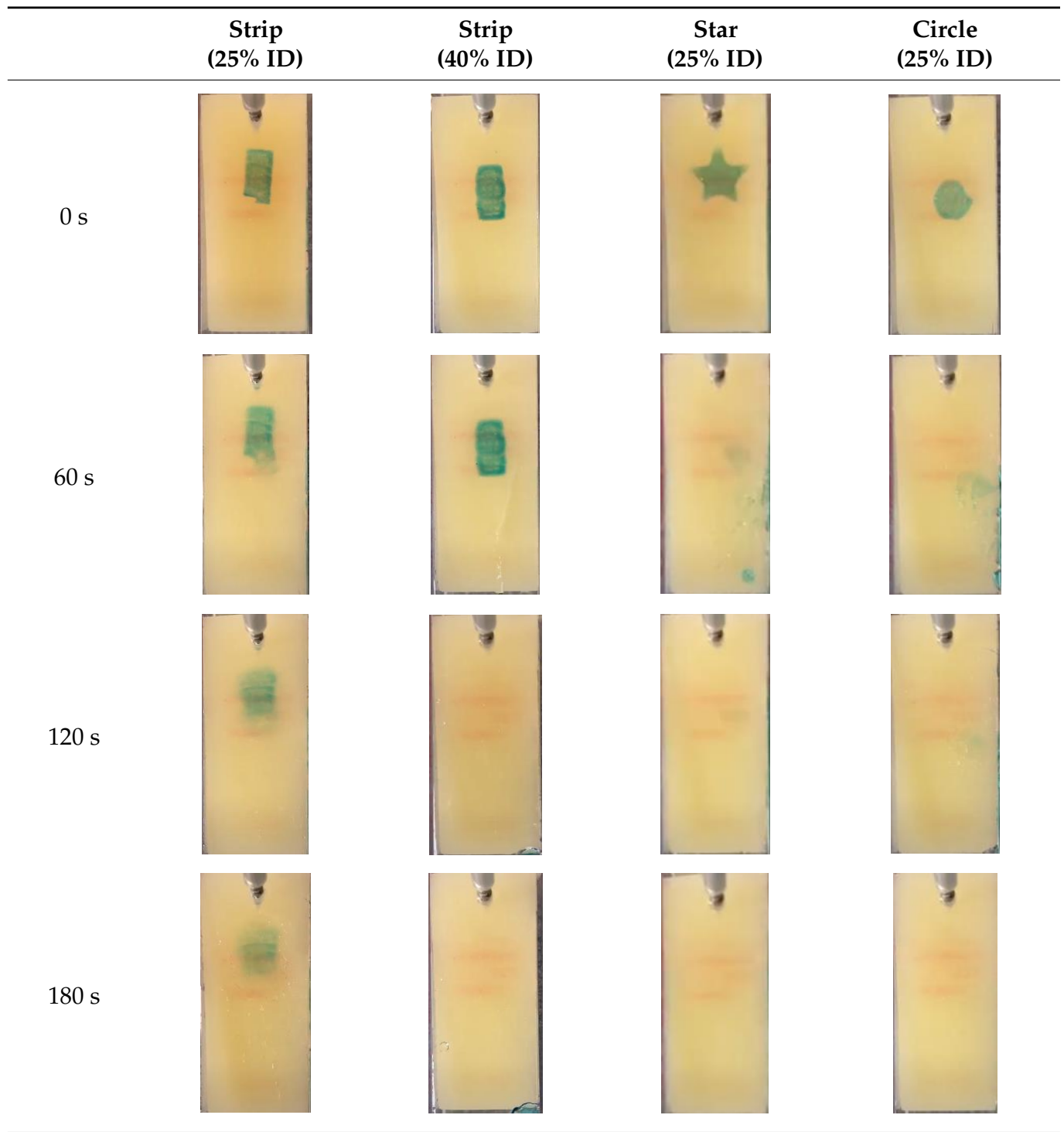

What is evident from both area-time profiles in Figures 4 and 5 is a temporary increase in area compared to the area at $t=0 \mathrm{~s}$. This increase reflects a temporary increase in blue dye over the synthetic tongue, as the film is being disintegrated, and subsequently releasing the blue dye. It is also evident that the temporary increase is followed by a catastrophic drop in area, reflecting the rapid removeable of the blue dye.

A modified Petri dish disintegration study was performed to validate the results from the OCM disintegration studies. The observed disintegration times of strip (154 $\pm 8 \mathrm{~s})$, star $(131 \pm 2 \mathrm{~s})$, and circle $(118 \pm 4 \mathrm{~s})$ geometries complied with Pharmacopeial requirements. As expected, the disintegration times were longer than that observed with the OCM, as the latter applied mechanical forces to accelerate disintegration (Figure 6). The modified petri dish results share a similar trend to the OCM observation, whereby both the star- and circle-shaped films presented with faster disintegration times. Further work is needed to explain this observation. 
(A)

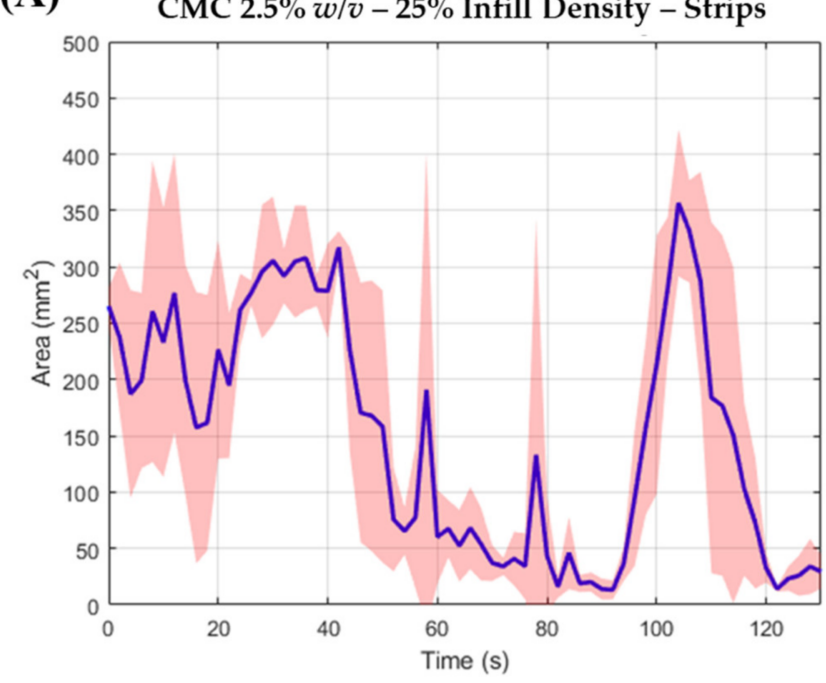

(B) CMC $2.5 \% w / v-40 \%$ Infill Density - Strips

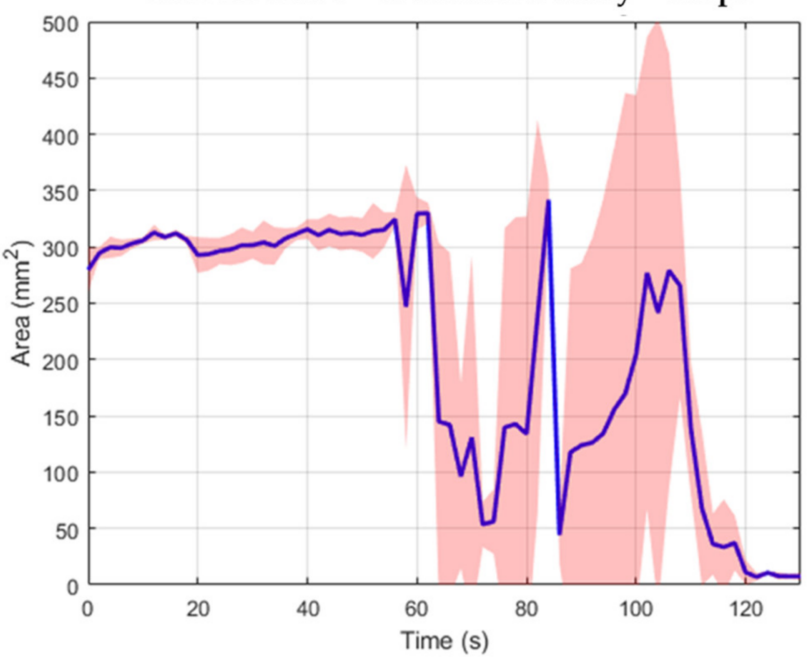

Figure 4. Mean top-down area-time profiles for (A) $25 \%$ and (B) $40 \%$ ID strip assessed in the OCM $(n=3)$.

(A)

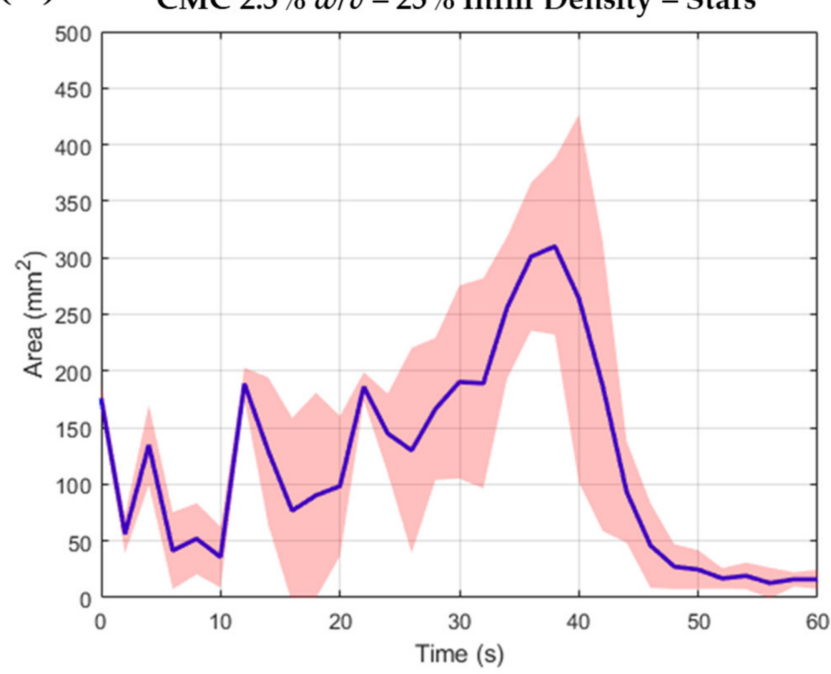

(B)

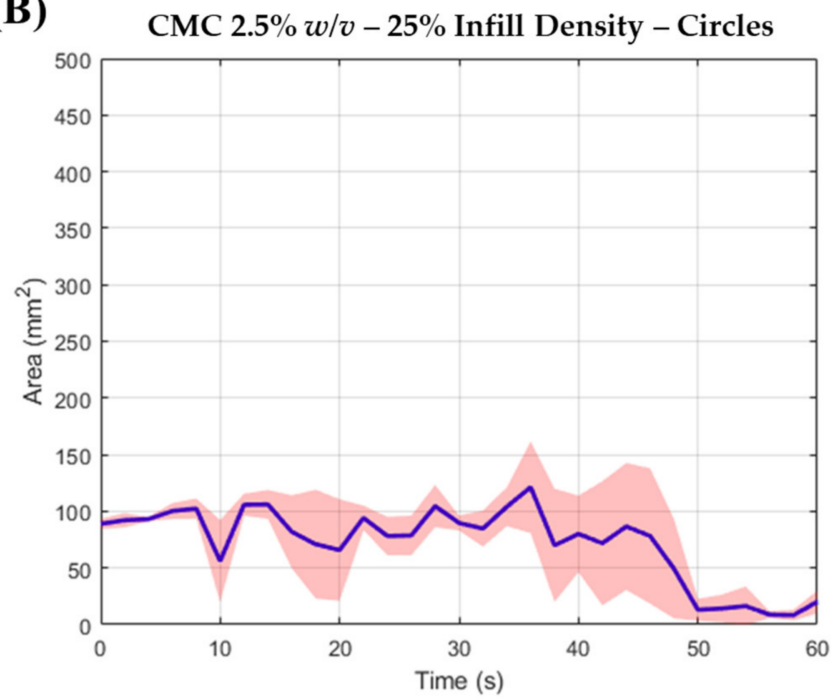

Figure 5. Mean top-down area-time profiles for (A) star and (B) circle geometries with 25\% ID, assessed in the OCM $(n=3)$.

The above results demonstrated that DIW is a suitable technology for ODF fabrication. The ideal CMC solution was $2.5 \mathrm{w} / \mathrm{v} \%$, which was found to possess rheological properties to achieve ODF structures with suitable resolutions. Figure 1 demonstrates different shapes that can be generated with this $\mathrm{CMC}$ concentration. In addition, the layer height and optimal ID were $3 \mathrm{~mm}$ and $25 \%$, respectively, which, post-drying, resulted in films with thickness below $100 \mu \mathrm{m}$. The film thicknesses reported herein are among the smallest values for 3D printed ODFs, such as those fabricated by fused deposition modelling (FDM), selective laser sintering and inkjet printing $[28,47]$. The advantage of DIW is that the process itself is rapid, and compared to FDM, feedstock preparation is rapid and does not require additional costly apparatus (i.e., hot-melt extruder). However, despite the aforementioned benefits, and the added advantages of rapid production and seamlessly producing different shapes, a limitation of DIW is the drying step. In this study, the films were left to dry overnight. This limitation affects other film fabrication technologies, including both solvent and film casting, but remains a subject of future work for the authors. 


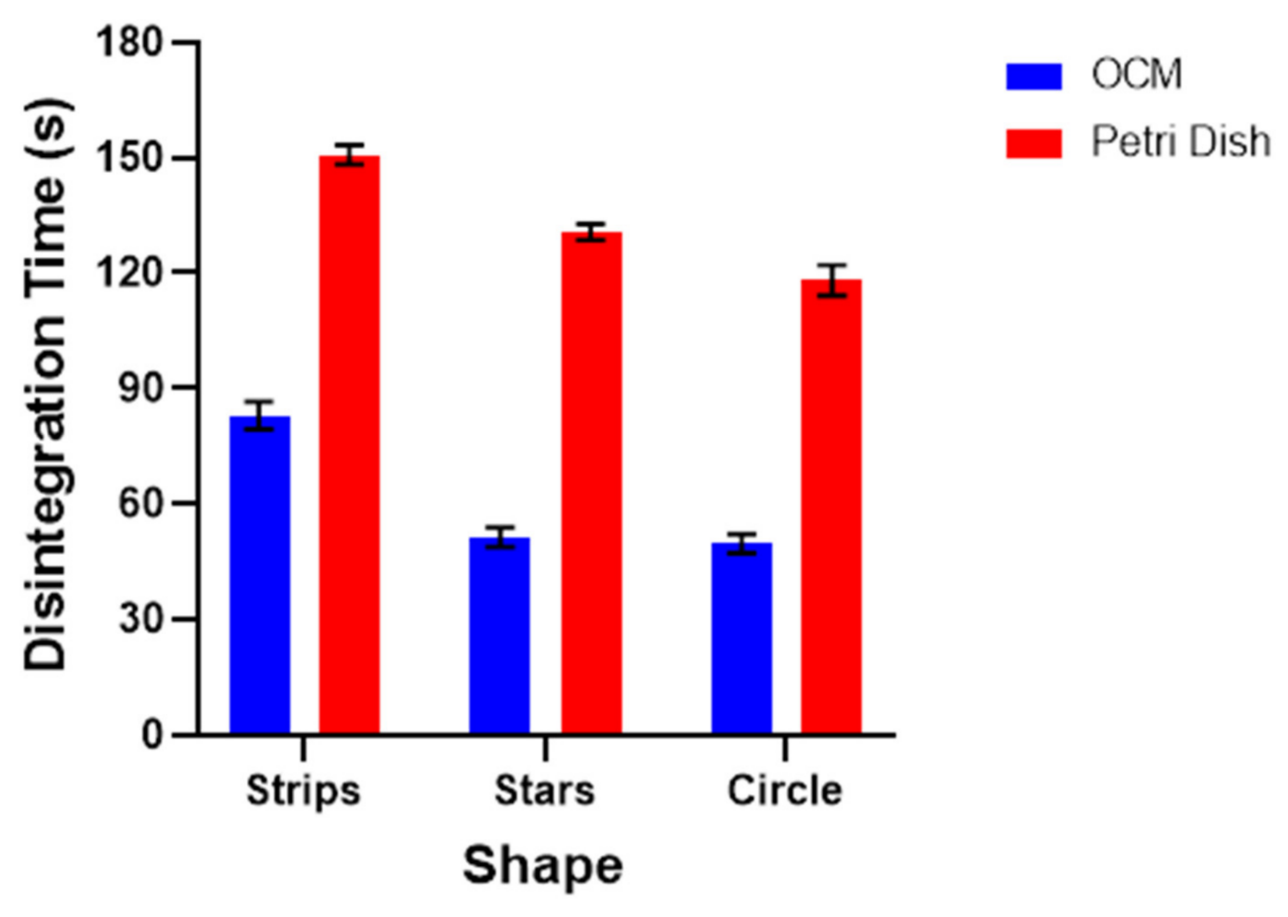

Figure 6. Disintegration time of films, as performed by both the OCM and modified petri dish.

\subsection{Drug and Dose Verification Using ML and NIR}

A limitation of translating 3D printing to clinical settings that has been previously highlighted is quality control of the drug product produced [28]. Ideally this process should be accurate as well as automated. NIR spectroscopy is an industry-standard analytical method for quality control. It provides rapid measurements and can differentiate between different materials. However, the analytical process is performed manually. An emerging technology capable of automating both drug and dose verification from NIR spectroscopy is ML. There are several tasks that can be achieved by ML, of which classification and regression are pertinent to this study. Classification tasks aim to classify similar groups together by identifying patterns in the dataset, whereas regression tasks aim to predict continuous variables. In the context of this study, a classification task was first used to determine the predictive performance of ML to identify which ODF is being analysed. To 'stress test' the ML model, two drugs with similar chemical structures were purposefully selected (i.e., caffeine and theophylline). The similarity score of these drugs was 0.95, according DrugBank. This was then followed by a regression task to quantify the concentration of API in the film, as quantified by HPLC (Table S1, see Supplementary Materials). The benefit of drug verification can be for either the operator or for internet of things (IoT). For the operator, an ML pipeline that automatically identifies which drug to perform the dose verification for will mitigate human error. For IoT applications, a database of formulation parameters can be seamlessly recorded.

\subsection{Classification of ODFs by API}

Classification can be achieved by either supervised or unsupervised learning. Supervised ML requires the output to be labelled, such that the machine learning technique (MLT) knows the target it should predict. Unsupervised on the other hand allows the MLT to classify the samples without needing pre-labelled data, essentially asking the MLT to inherently detect patterns. The advantage of unsupervised learning is that it obviates the need for a user to pre-label the data, which in a dataset consisting of a large number of samples, can be time consuming. In this study, both supervised and unsupervised classification were explored to determine the optimal model.

Principal component analysis (PCA) is a common unsupervised learner with multiple applications in ML [48]. PCA was used to decompose the NIR spectra, consisting of 16,595 
dimensions, to 3 dimensions that can be visualised. The NIR spectra were grouped by their API (i.e., paracetamol, caffeine, and theophylline) as well as the API-free films, which are referred to as pristine. The results of decomposing the NIR spectra using PCA are presented in Figure 7. PCA was able to approximate the different APIs into clusters to some extent. For example, paracetamol spectra and caffeine spectra were found to cluster to the right and left, respectively, in the three-dimensional space; thus, if a new ODF spectrum fell within either of these clusters then there is a high degree of certainty that it would contain the respective API. However, the clusters were found to overlap, and thus a $100 \%$ accuracy could not be achieved. This would pose a challenge if PCA was paired with a clustering algorithm which seeks to automatically detect clusters in datasets. To illustrate this hypothesis, three unsupervised clustering algorithms were used: k-means, density-based spatial clustering of applications with noise (DBSCAN) and hierarchical clustering. Figure 8 illustrates a two-component PCA plot, as well as the results of the three clustering algorithms. It can be observed that while all three clustering algorithms were able to successfully cluster the lower-right cluster (i.e., paracetamol), an exact match to the true clusters was not obtained for the other APIs.

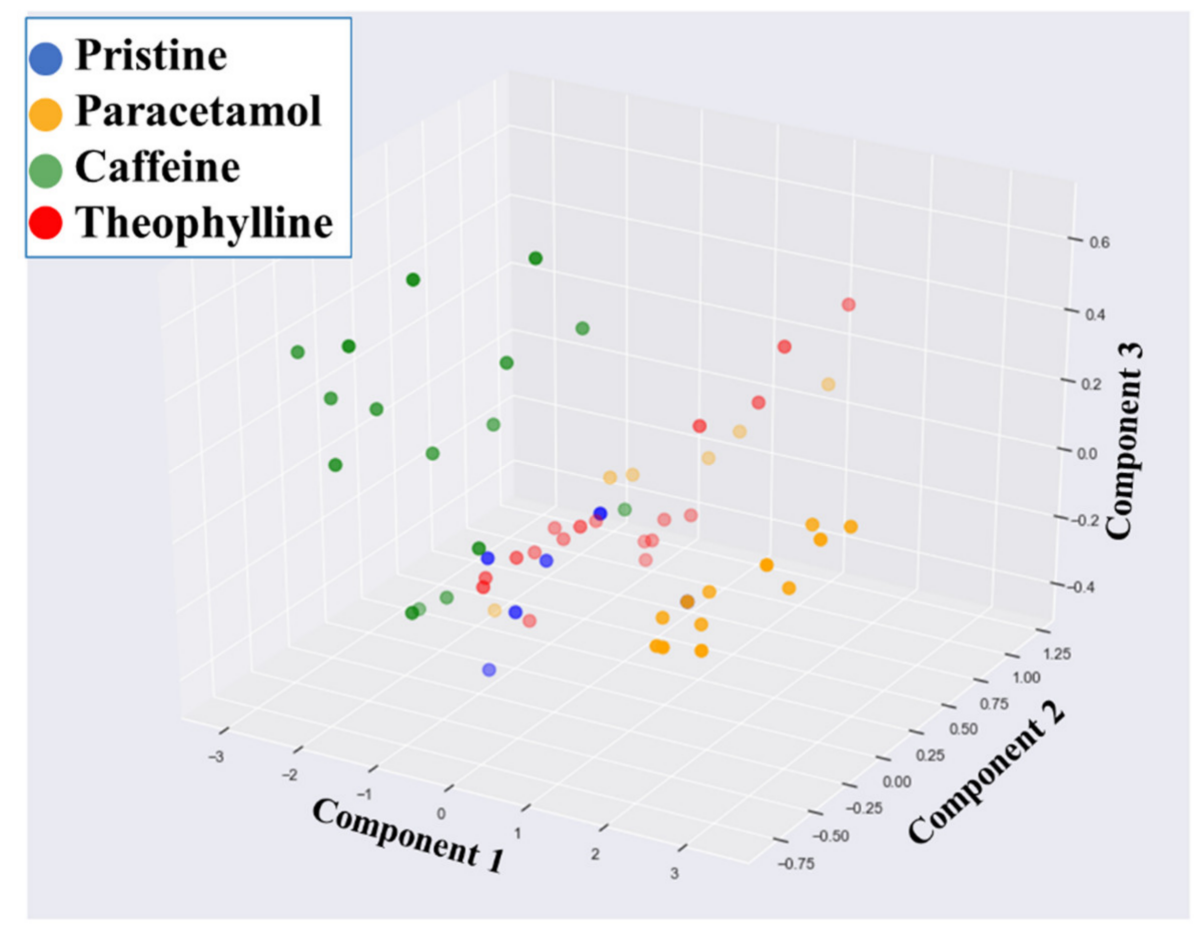

Figure 7. PCA decomposition of the raw NIR spectra to three dimensions (PCA 1-1st principal component 1; PCA 2-2nd principal component).

In addition to PCA, $t$-distributed stochastic neighbour embedding ( $t$-sne) and kernel PCA (kPCA) were also applied to the raw NIR spectra. One limitation with PCA is that it is a linear transformer, and hence assumes that the data possess a linear relationship. Both t-sne and kPCA are able to decompose the dataset using non-linear transformation. However, a similar finding to PCA was revealed, in that an exact clustering was not obtained (Figure 9). Therefore, it was concluded that PCA, t-sne and kPCA were unable to classify the APIs. 

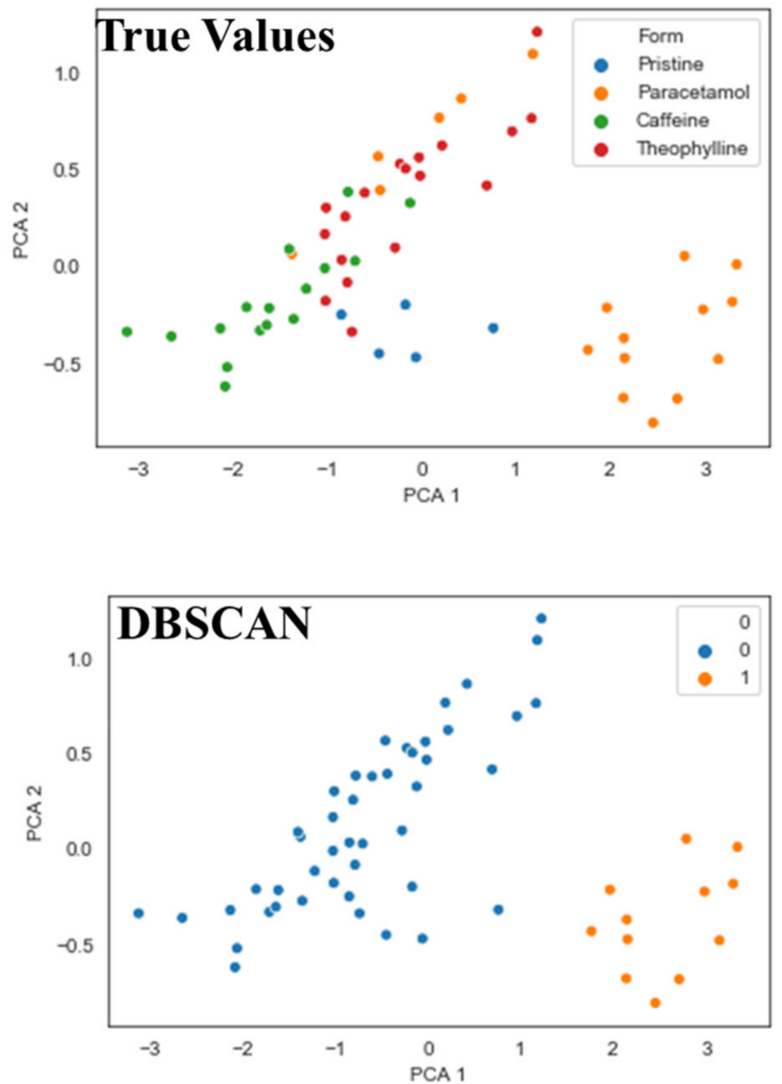
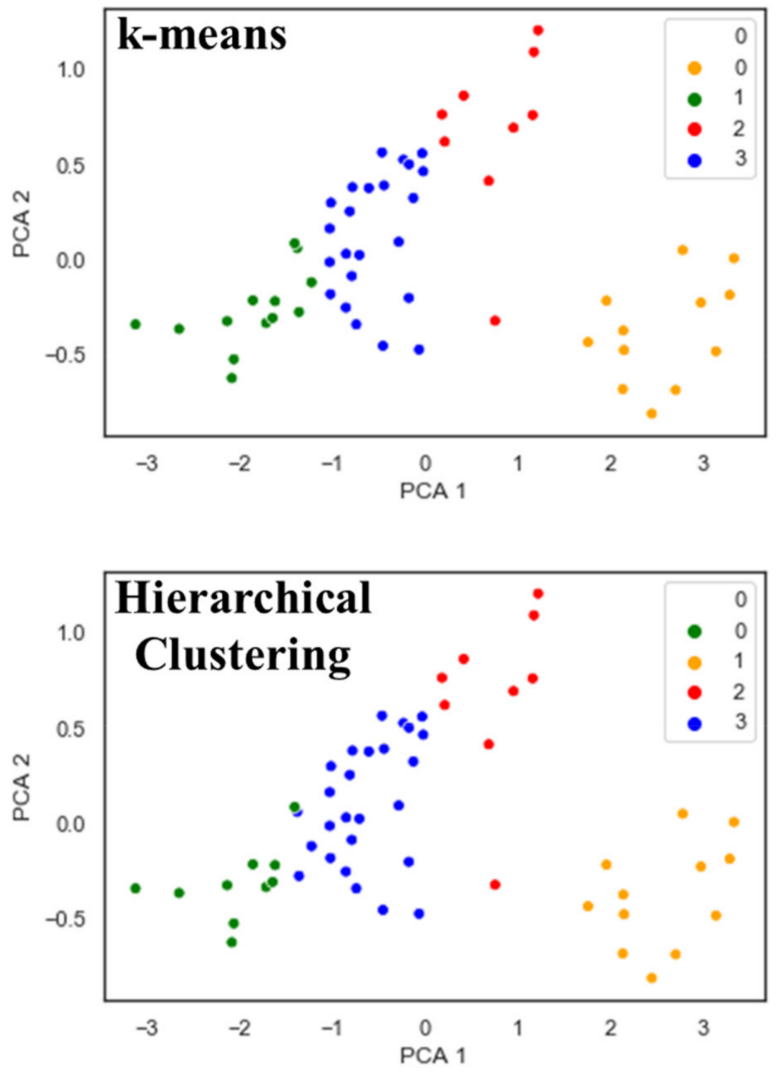

Figure 8. Two component PCA of the raw NIR spectra. PCA resulted in overlaps between clusters, which k-means, DBSCAN and hierarchical clustering were unable to exactly replicate. k-means and hierarchical clustering inherently identified four different clusters (clusters 0,1,2, and 3); however, they were not an exact match to the true values. DBSCAN was only able to detect two clusters (0 and 1) (PCA 1-1st principal component 1; PCA 2-2nd principal component).
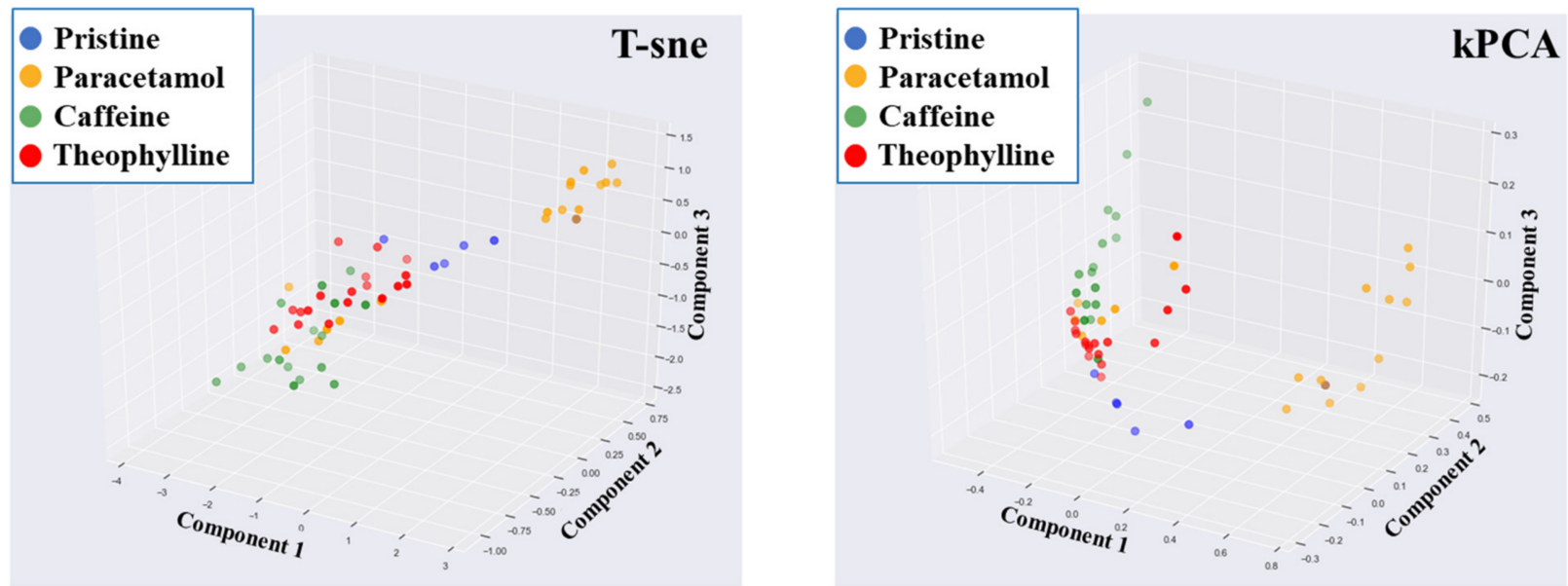

Figure 9. Non-linear transformation of the raw NIR spectra using T-sne and kPCA (kPCA1-1st principal component; kPCA2-2nd principal component).

As unsupervised learning was unsuccessful, supervised learning was then employed. A common supervised learner is linear discriminant analysis (LDA). This algorithm is similar to PCA; however, with the use of a pre-labelled dataset, it seeks to maximise the distance between the classes [49]. For this study, LDA was more effective in classifying the spectra according to their API constituent. Analysing the raw spectra using LDA yielded an improved separation between the different APIs (Figure 10) compared to the three 
unsupervised learners used. Unique clustering groups were observed for the paracetamolcontaining films, with a minor overlap between the caffeine- and theophylline-containing films, as well as minor overlaps between the pristine and caffeine-containing films. A simple pre-processing step using a Savitzky-Golay filter resulted in complete separation between the different ODFs (Figure 10). Therefore, an ML pipeline of Savitzky-Golay pre-processing of NIR with LDA was found to be the most efficient pipeline for this study. To demonstrate the efficiency of this approach, the NIR dataset was split into 70:30, where the $70 \%$ was used to develop a predictive model (i.e., the training set), and the other $30 \%$ was used to evaluate the accuracy of the model (i.e., the testing set). A $100 \%$ prediction accuracy was achieved using the pre-processed spectra, whereas the accuracy with using the raw spectra was $88.9 \%$. A confusion matrix of the result (Figure 11) revealed that two pristine spectra were predicted as caffeine, which is understandable as both were found to form clusters in similar data space.
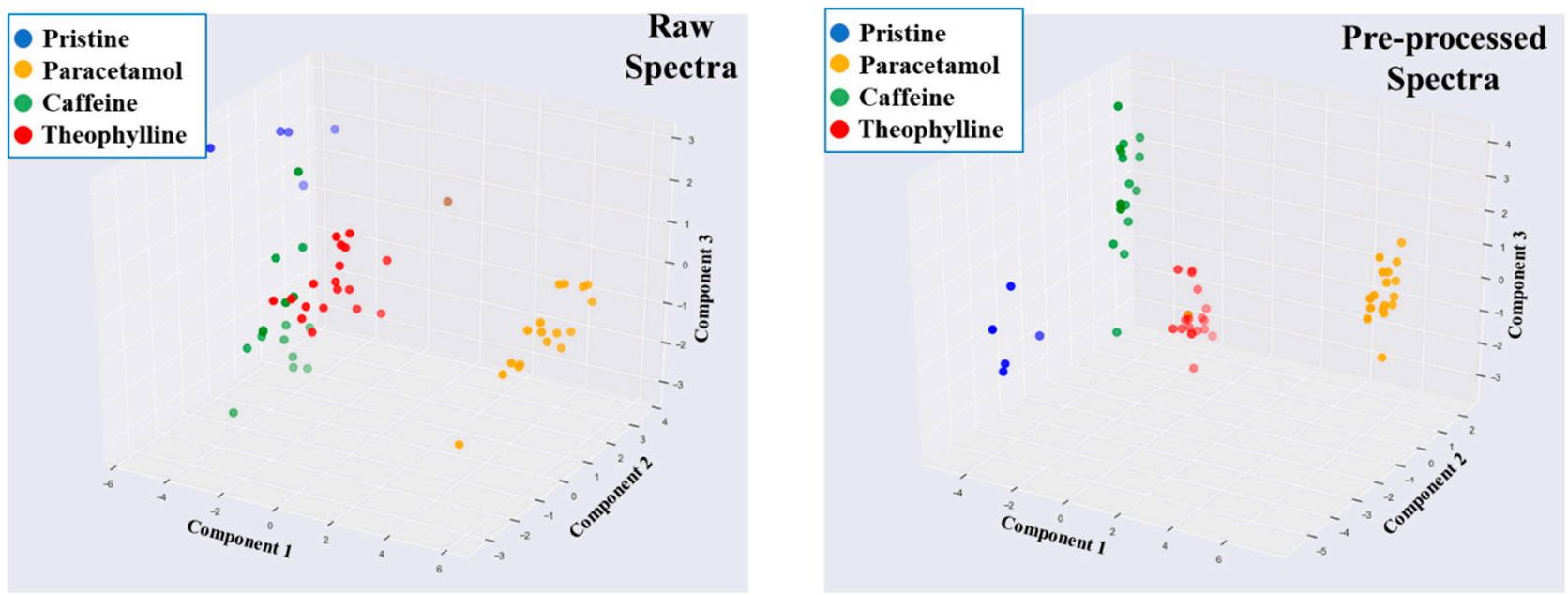

Figure 10. LDA classification of the raw and pre-processed spectra. Pre-processing of the NIR spectra yielded the highest accurate clustering.
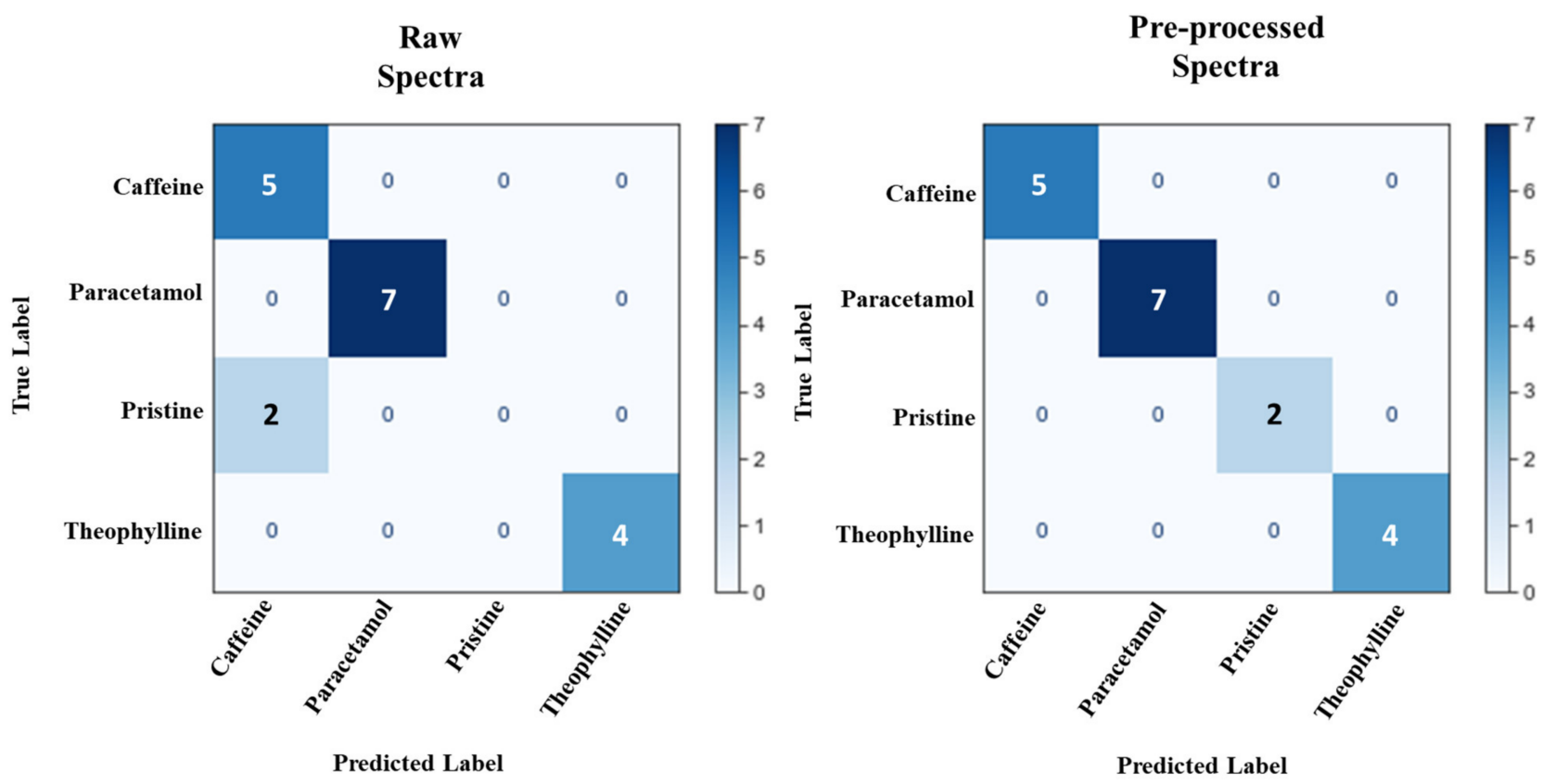

Figure 11. Confusion matrix depicting the results of the LDA prediction for both the raw and pre-processed NIR spectra. 


\subsection{Regression for Quantifying API Dose}

Following the successful clustering of films according to their API constituent, a regression model was developed to predict the API dose for each class. Partial least squares (PLS) was employed in this study. PLS is a common supervised learner suited for NIR owing to its ability to handle datasets where the number of dimensions are far greater than the sample size [50,51]. Applying PLS to the aforementioned ML pipeline, it was able to achieve an $\mathrm{R}^{2}$ value of above 0.99 for each of the classes. Each API class included the spectra of pristine as $0 w / w \%$ API content. To test the accuracy of PLS, the dataset was split into training and testing, using a 75:25 split, which resulted in a predicted $\mathrm{R}^{2}$ of above 0.96 for each of the API class. Splitting the data into training and testing allows for the latter to essentially behave as 'blind data', which provides a more robust evaluation of the ML model (Figure 12).

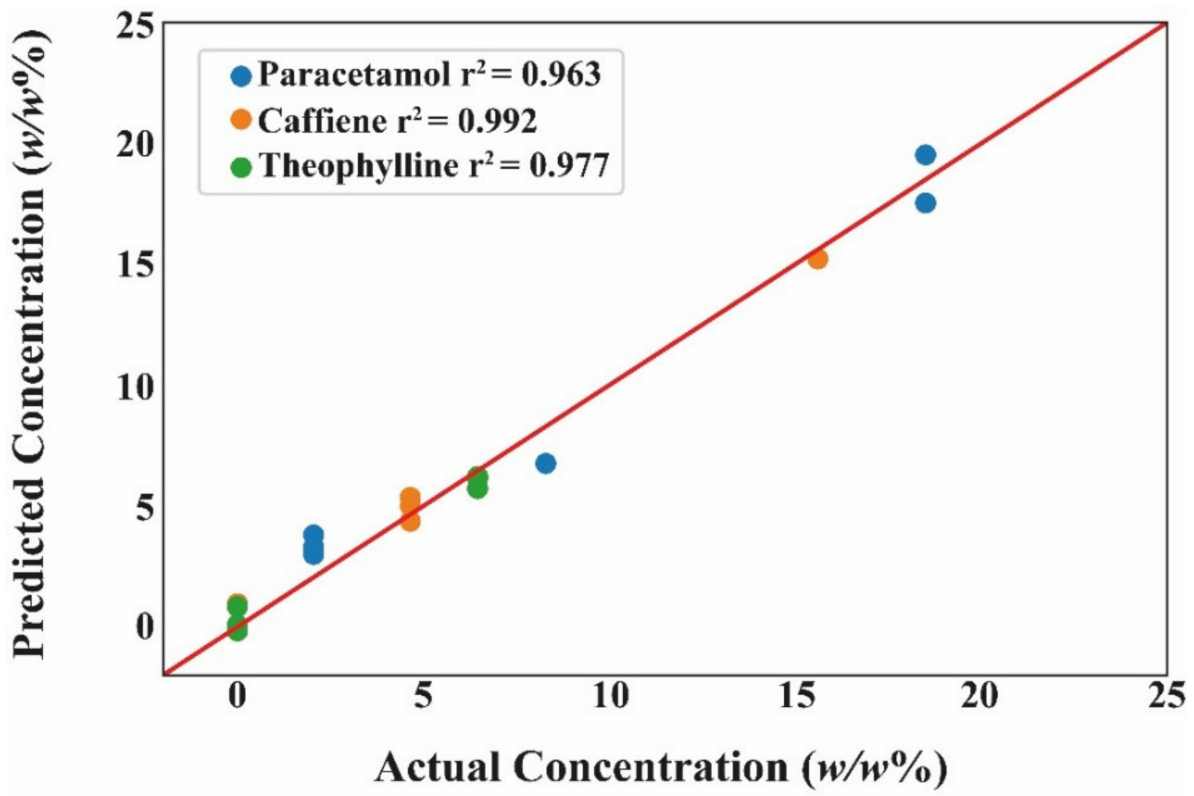

Figure 12. Comparing the predicted to the actual drug concentration on the test dataset for each API. The results are of the pre-processed NIR-ML data using partial least squares.

\subsection{Realising the Benefits of Digital Technologies}

The study began by considering the tools needed to further enhance ODF development, with the aim of making the manufacturing process suitable for personalisation and on-demand production. It transpired that digital technologies possessed the tools needed to deliver this goal [52]. At the research setting, 3D printing, MV and ML were found to perform tasks whilst minimising human supervision. The former allowed multiple films to be produced once the process was started, whereas contemporary fabrication methods require each film to be manually cut. Similarly, MV was able to determine the disintegration times of films, which currently is performed through constant observation by the researcher. Lastly, ML was able to interpret NIR spectra, replacing the need for human interpretation of the individual peaks. It is also worth remarking that $3 \mathrm{D}$ printing and MV provided digital precision in the order of micrometres and milliseconds, respectively. ML on the other hand was found to rapidly complete the drug and dose verification task. The predictions made with the proposed ML pipeline took $0.091 \mathrm{~s}$ for LDA to be trained, and less than $0.001 \mathrm{~s}$ to classify one NIR spectrum. For PLS, the training and prediction per sample were 0.015 and $0.001 \mathrm{~s}$, respectively. Moreover, in clinical settings, 3D printing and ML will ensure tasks can be replicated whilst diminishing the need for onsite expert supervision, which in turn will reduce expenses. The current work revealed that unsupervised algorithms were not $100 \%$ accurate in differentiating between the different APIs, particularly between caffeine 
and theophylline. Consequently, there will be a need for an expert to label the NIR spectra for training, which can be done offsite. Society, including both the pharmaceutics and healthcare sectors, is currently moving towards the next industrial revolution, Industry 4.0, where all three digital technologies are implicated therein [52]. The present study provides a strong validation for the use of digital technologies, showcasing their potential for an automated and reliable ODF workflow.

\section{Conclusions}

The present study confirmed that DIW was able to produce ODFs of bespoke shapes and with suitable disintegrating times. The ideal CMC concentration and infill were $2.5 \%$ $w / w$ and $25 \%$, respectively, which resulted in ODFs disintegrating far within the European Pharmacopeia specified $3 \mathrm{~min}$. Three different API-containing films were fabricated (paracetamol, caffeine, and theophylline), where for each API category three varying concentrations of between $0-20 \% w / w$ were prepared. In addition, a robust ML pipeline was proposed for verifying both the drug and dose of ODFs. Both unsupervised and supervised learning were evaluated for drug verification, where it was discovered that supervised learning outperformed three different unsupervised learners. LDA was able to achieve drug verification accuracies of $88.9 \%$ and $100 \%$ with raw and pre-processed NIR spectra, respectively. Dose verification for all three API ODFs categories yielded high accuracies, with $\mathrm{r}^{2}$ values above 0.96 . The study also revealed that the proposed ML pipeline was computationally undemanding, and suitable for scale-up with prediction times of $0.001 \mathrm{~s}$ per sample. Therefore, NIR spectroscopy and ML were confirmed to be suitable technologies for rapid drug and dose verification. Future work will concentrate in minimising the drying step of ODFs prepared by DIW.

Supplementary Materials: The following are available online at https:/ /www.mdpi.com/article/10 .3390 / pharmaceutics13122187/s1, Figure S1: Representative low, medium and high-concentration drug-loaded film, Table S1: API concentration as determined by HPLC.

Author Contributions: Conceptualisation, M.E., S.G., A.W.B., M.O.; Methodology, C.S.O., M.E., N.D.; Formal Analysis, C.S.O., M.E., N.D.; Resources, S.G., A.W.B., M.O.; Writing-Original Draft Preparation, C.S.O., N.D.; Writing-Review and Editing, M.E., S.G., A.W.B., M.O.; Visualisation, C.S.O., M.E., N.D.; Supervision, S.G., A.W.B., M.O.; Funding Acquisition, S.G., A.W.B., M.O. All authors have read and agreed to the published version of the manuscript.

Funding: C.S.O., M.E. and N.D. were funded by the Engineering and Physical Sciences Research Council (EPSRC) [Grant Numbers: EP/S023054/1, EP/S009000/1, EP/L01646X/1].

Institutional Review Board Statement: Not applicable.

Informed Consent Statement: Not applicable.

Data Availability Statement: The data presented in this study are available upon request from the corresponding author.

Conflicts of Interest: The authors declare that they have no known competing financial interests or personal relationships that could have appeared to influence the work reported in this paper.

\section{References}

1. Liu, J.; Guan, J.; Wan, X.; Shang, R.; Shi, X.; Fang, L.; Liu, C. The Improved Cargo Loading and Physical Stability of Ibuprofen Orodispersible Film: Molecular Mechanism of Ion-Pair Complexes on Drug-Polymer Miscibility. J. Pharm. Sci. 2019, 109, 1356-1364. [CrossRef] [PubMed]

2. Boulaiz, H.; Ramos, M.C.; Grinan-Lison, C.; Garcia-Rubino, M.E.; Vicente, F.; Marchal, J.A. What's new in the diagnosis of pancreatic cancer: A patent review (2011-present). Expert Opin. Ther. Pat. 2017, 27, 1319-1328. [CrossRef]

3. Wang, B.; Yang, L.; Luo, C.; Wang, Y.; Wang, H.; Chen, F.; Xiang, X. Development, In Vitro and In Vivo Evaluation of Racecadotril Orodispersible Films for Pediatric Use. AAPS PharmSciTech 2021, 22, 1-13. [CrossRef] [PubMed]

4. Gupta, M.S.; Kumar, T.P.; Davidson, R.; Kuppu, G.R.; Pathak, K.; Gowda, D.V. Printing Methods in the Production of Orodispersible Films. AAPS PharmSciTech 2021, 22, 1-17. [CrossRef] [PubMed] 
5. Seoane-Viaño, I.; Trenfield, S.J.; Basit, A.W.; Goyanes, A. Translating 3D printed pharmaceuticals: From hype to real-world clinical applications. Adv. Drug Deliv. Rev. 2021, 174, 553-575. [CrossRef] [PubMed]

6. Trenfield, S.J.; Xian Tan, H.; Awad, A.; Buanz, A.; Gaisford, S.; Basit, A.W.; Goyanes, A. Track-and-trace: Novel anti-counterfeit measures for 3D printed personalized drug products using smart material inks. Int. J. Pharm. 2019, 567, 118443. [CrossRef] [PubMed]

7. Seoane-Viaño, I.; Gómez-Lado, N.; Lázare-Iglesias, H.; García-Otero, X.; Antúnez-López, J.R.; Ruibal, Á.; Varela-Correa, J.J.; Aguiar, P.; Basit, A.W.; Otero-Espinar, F.J.; et al. 3D Printed Tacrolimus Rectal Formulations Ameliorate Colitis in an Experimental Animal Model of Inflammatory Bowel Disease. Biomedicines 2020, 8, 563. [CrossRef] [PubMed]

8. Xu, X.; Pose-Boirazian, T.; Eibes, G.; McCoubrey, L.E.; Martínez-Costas, J.; Gaisford, S.; Goyanes, A.; Basit, A.W. A customizable 3D printed device for enzymatic removal of drugs in water. Water Res. 2021, 208, 117861. [CrossRef] [PubMed]

9. Xu, X.; Seijo-Rabina, A.; Awad, A.; Rial, C.; Gaisford, S.; Basit, A.W.; Goyanes, A. Smartphone-enabled 3D printing of medicines. Int. J. Pharm. 2021, 609, 121199. [CrossRef] [PubMed]

10. Domínguez-Robles, J.; Diaz-Gomez, L.; Utomo, E.; Shen, T.; Picco, C.J.; Alvarez-Lorenzo, C.; Concheiro, A.; Donnelly, R.F.; Larrañeta, E. Use of 3D Printing for the Development of Biodegradable Antiplatelet Materials for Cardiovascular Applications. Pharmaceuticals 2021, 14, 921. [CrossRef]

11. Falahati, M.; Ahmadvand, P.; Safaee, S.; Chang, Y.-C.; Lyu, Z.; Chen, R.; Li, L.; Lin, Y. Smart polymers and nanocomposites for 3D and 4D printing. Mater. Today 2020, 40, 215-245. [CrossRef]

12. Jamróz, W.; Kurek, M.; Lyszczarz, E.; Szafraniec-Szczęsny, J.; Knapik-Kowalczuk, J.; Syrek, K.; Paluch, M.; Jachowicz, R. 3D printed orodispersible films with Aripiprazole. Int. J. Pharm. 2017, 533, 413-420. [CrossRef]

13. Sjöholm, E.; Sandler, N. Additive manufacturing of personalized orodispersible warfarin films. Int. J. Pharm. 2019, 564, 117-123. [CrossRef]

14. Kiefer, O.; Fischer, B.; Breitkreutz, J. Fundamental Investigations into Metoprolol Tartrate Deposition on Orodispersible Films by Inkjet Printing for Individualised Drug Dosing. Pharmaceutics 2021, 13, 247. [CrossRef]

15. Thabet, Y.; Lunter, D.; Breitkreutz, J. Continuous inkjet printing of enalapril maleate onto orodispersible film formulations. Int. J. Pharm. 2018, 546, 180-187. [CrossRef] [PubMed]

16. Edinger, M.; Bar-Shalom, D.; Sandler, N.; Rantanen, J.; Genina, N. QR encoded smart oral dosage forms by inkjet printing. Int. J. Pharm. 2018, 536, 138-145. [CrossRef] [PubMed]

17. Cho, H.-W.; Baek, S.-H.; Lee, B.-J.; Jin, H.-E. Orodispersible Polymer Films with the Poorly Water-Soluble Drug, Olanzapine: Hot-Melt Pneumatic Extrusion for Single-Process 3D Printing. Pharmaceutics 2020, 12, 692. [CrossRef]

18. Panraksa, P.; Qi, S.; Udomsom, S.; Tipduangta, P.; Rachtanapun, P.; Jantanasakulwong, K.; Jantrawut, P. Characterization of Hydrophilic Polymers as a Syringe Extrusion 3D Printing Material for Orodispersible Film. Polymers 2021, 13, 3454. [CrossRef]

19. Karavasili, C.; Eleftheriadis, G.K.; Gioumouxouzis, C.; Andriotis, E.G.; Fatouros, D.G. Mucosal drug delivery and 3D printing technologies: A focus on special patient populations. Adv. Drug Deliv. Rev. 2021, 176, 113858. [CrossRef]

20. Rahman, J.; Quodbach, J. Versatility on demand-The case for semi-solid micro-extrusion in pharmaceutics. Adv. Drug Deliv. Rev. 2021, 172, 104-126. [CrossRef] [PubMed]

21. Economidou, S.N.; Uddin, J.; Marques, M.J.; Douroumis, D.; Sow, W.T.; Li, H.; Reid, A.; Windmill, J.F.; Podoleanu, A. A novel 3D printed hollow microneedle microelectromechanical system for controlled, personalized transdermal drug delivery. Addit. Manuf. 2020, 38, 101815. [CrossRef]

22. Nazir, A.; Azhar, A.; Nazir, U.; Liu, Y.-F.; Qureshi, W.S.; Chen, J.-E.; Alanazi, E. The rise of 3D Printing entangled with smart computer aided design during COVID-19 era. J. Manuf. Syst. 2021, 60, 774-786. [CrossRef] [PubMed]

23. Beer, N.; Hegger, I.; Kaae, S.; De Bruin, M.L.; Genina, N.; Alves, T.L.; Hoebert, J.; Sporrong, S.K. Scenarios for 3D printing of personalized medicines-A case study. Explor. Res. Clin. Soc. Pharm. 2021, 4, 100073. [CrossRef]

24. European Medicines Agency. Guideline on Real Time Release Testing (formerly Guideline on Parametric Release). Available online: https:/ / www.ema.europa.eu/en/real-time-release-testing (accessed on 13 December 2021).

25. Trenfield, S.J.; Goyanes, A.; Telford, R.; Wilsdon, D.; Rowland, M.; Gaisford, S.; Basit, A.W. 3D printed drug products: Nondestructive dose verification using a rapid point-and-shoot approach. Int. J. Pharm. 2018, 549, 283-292. [CrossRef]

26. Melocchi, A.; Briatico-Vangosa, F.; Uboldi, M.; Parietti, F.; Turchi, M.; von Zeppelin, D.; Maroni, A.; Zema, L.; Gazzaniga, A.; Zidan, A. Quality considerations on the pharmaceutical applications of fused deposition modeling 3D printing. Int. J. Pharm. 2020, 592, 119901. [CrossRef] [PubMed]

27. Trenfield, S.J.; Tan, H.X.; Goyanes, A.; Wilsdon, D.; Rowland, M.; Gaisford, S.; Basit, A.W. Non-destructive dose verification of two drugs within 3D printed polyprintlets. Int. J. Pharm. 2020, 577, 119066. [CrossRef] [PubMed]

28. McCoubrey, L.E.; Gaisford, S.; Orlu, M.; Basit, A.W. Predicting drug-microbiome interactions with machine learning. Biotechnol. Adv. 2021, 107797. [CrossRef]

29. Elbadawi, M.; McCoubrey, L.E.; Gavins, F.K.; Ong, J.J.; Goyanes, A.; Gaisford, S.; Basit, A.W. Disrupting 3D printing of medicines with machine learning. Trends Pharmacol. Sci. 2021, 42, 745-757. [CrossRef]

30. McCoubrey, L.; Elbadawi, M.; Orlu, M.; Gaisford, S.; Basit, A. Machine Learning Uncovers Adverse Drug Effects on Intestinal Bacteria. Pharmaceutics 2021, 13, 1026. [CrossRef] [PubMed]

31. Wang, F.; Elbadawi, M.; Tsilova, S.L.; Gaisford, S.; Basit, A.W.; Parhizkar, M. Machine learning to empower electrohydrodynamic processing. Mater. Sci. Eng. C 2021, 132, 112553. [CrossRef] 
32. Baker, D.J. Artificial Intelligence: The Future Landscape of Genomic Medical Diagnosis: Dataset, In Silico Artificial Intelligent Clinical Information, and Machine Learning Systems. In Human Genome Informatics. Translating Genes Into Health; Lambert, C.G., Baker, D.J., Patrinos, G.P., Eds.; Elsevier Inc.: Amsterdam, The Netherlands, 2018; pp. 223-267.

33. Xianyu, Y.; Wang, Q.; Chen, Y. Magnetic particles-enabled biosensors for point-of-care testing. TrAC Trends Anal. Chem. 2018, 106, 213-224. [CrossRef]

34. Popova, M.; Isayev, O.; Tropsha, A. Deep reinforcement learning for de novo drug design. Sci. Adv. 2018, 4, eaap7885. [CrossRef] [PubMed]

35. Hosny, A.; Parmar, C.; Quackenbush, J.; Schwartz, L.H.; Aerts, H.J.W.L. Artificial intelligence in radiology. Nat. Rev. Canc. 2018, 18, 500-510. [CrossRef] [PubMed]

36. Han, R.; Xiong, H.; Ye, Z.; Yang, Y.; Huang, T.; Jing, Q.; Lu, J.; Pan, H.; Ren, F.; Ouyang, D. Predicting physical stability of solid dispersions by machine learning techniques. J. Control. Release 2019, 311-312, 16-25. [CrossRef] [PubMed]

37. Harrer, S.; Shah, P.; Antony, B.; Hu, J. Artificial Intelligence for Clinical Trial Design. Trends Pharmacol. Sci. 2019, 40, 577-591. [CrossRef] [PubMed]

38. Elbadawi, M.; Castro, B.M.; Gavins, F.K.; Ong, J.J.; Gaisford, S.; Pérez, G.; Basit, A.W.; Cabalar, P.; Goyanes, A. M3DISEEN: A novel machine learning approach for predicting the 3D printability of medicines. Int. J. Pharm. 2020, 590, 119837. [CrossRef] [PubMed]

39. Castro, B.M.; Elbadawi, M.; Ong, J.J.; Pollard, T.; Song, Z.; Gaisford, S.; Pérez, G.; Basit, A.W.; Cabalar, P.; Goyanes, A. Machine learning predicts 3D printing performance of over 900 drug delivery systems. J. Control. Release 2021, 337, 530-545. [CrossRef] [PubMed]

40. Redfearn, A.; Hanson, B. A Mechanical Simulator of Tongue-Palate Compression to Investigate the Oral Flow of Non-Newtonian Fluids. IEEE/ASME Trans. Mechatron. 2018, 23, 958-965. [CrossRef]

41. Desai, N.; Redfearn, A.; MacLeod, G.; Tuleu, C.; Hanson, B.; Orlu, M. How Do Orodispersible Tablets Behave in an In Vitro Oral Cavity Model: A Pilot Study. Pharmaceutics 2020, 12, 651. [CrossRef] [PubMed]

42. Levy, G. Viscosity-Stability of Aqueous Solutions of Certain Hydrophilic Polymers. J. Pharm. Sci. 1961, 50, 429-435. [CrossRef] [PubMed]

43. Benchabane, A.; Bekkour, K. Rheological properties of carboxymethyl cellulose (CMC) solutions. Colloid Polym. Sci. 2008, 286, 1173-1180. [CrossRef]

44. Elbadawi, M. Polymeric Additive Manufacturing: The Necessity and Utility of Rheology. In Polymer Rheology; Rivera-Armenta, L., Salazar Cruz, B.A., Eds.; IntechOpen: London, UK, 2018; pp. 43-65.

45. European Pharmacopoeia Commision. European Pharmacopoeia 10.0 Oromucosal Preparations; European Directorate for the Quality of Medicines \& Healthcare (EDQM): Strasbourg, France, 2020; Volume 939, pp. 323-324.

46. Ehtezazi, T.; Algellay, M.; Islam, Y.; Roberts, M.; Dempster, N.M.; Sarker, S.D. The Application of 3D Printing in the Formulation of Multilayered Fast Dissolving Oral Films. J. Pharm. Sci. 2018, 107, 1076-1085. [CrossRef] [PubMed]

47. Cichocki, A. Unsupervised Learning Algorithms and Latent Variable Models: PCA/SVD, CCA/PLS, ICA, NMF, etc. In Academic Press Library in Signal Processing; Diniz, P.S.R., Suykens, J.A.K., Chellappa, R., Theodoridis, S., Eds.; Elsevier Inc.: Amsterdam, The Netherlands, 2014; Volume 1, pp. 1151-1238.

48. Moncayo, S.; Manzoor, S.; Navarro-Villoslada, F.; Caceres, J. Evaluation of supervised chemometric methods for sample classification by Laser Induced Breakdown Spectroscopy. Chemom. Intell. Lab. Syst. 2015, 146, 354-364. [CrossRef]

49. Clark, M.; Cramer, R.D. The Probability of Chance Correlation Using Partial Least Squares (PLS). Quant. Struct. Relationships 1993, 12, 137-145. [CrossRef]

50. Elbadawi, M.; McCoubrey, L.E.; Gavins, F.K.; Ong, J.J.; Goyanes, A.; Gaisford, S.; Basit, A.W. Harnessing artificial intelligence for the next generation of 3D printed medicines. Adv. Drug Deliv. Rev. 2021, 175, 113805. [CrossRef] [PubMed]

51. Raijada, D.; Wac, K.; Greisen, E.; Rantanen, J.; Genina, N. Integration of personalized drug delivery systems into digital health. Adv. Drug Deliv. Rev. 2021, 176, 113857. [CrossRef]

52. Awad, A.; Trenfield, S.J.; Pollard, T.D.; Ong, J.J.; Elbadawi, M.; McCoubrey, L.E.; Goyanes, A.; Gaisford, S.; Basit, A.W. Connected healthcare: Improving patient care using digital health technologies. Adv. Drug Deliv. Rev. 2021, 178, 113958. [CrossRef] [PubMed] 